\title{
Rapid angular expansion of the ionized core of CRL 618
}

\author{
D. Tafoya ${ }^{1}$, L. Loinard ${ }^{2}$, J. P. Fonfría ${ }^{3}$, W. H. T. Vlemmings ${ }^{1}$, I. Martí-Vidal ${ }^{1}$, and G. Pech ${ }^{2}$
}

\author{
${ }^{1}$ Chalmers University of Technology, Onsala Space Observatory, 43992 Onsala, Sweden \\ e-mail: tafoya@chalmers.se \\ 2 Centro de Radioastronomía y Astrofísica, UNAM, Apdo. Postal 3-72 (Xangari), 58089 Morelia, Michoacán, México \\ 3 Departamento de Estrellas y Medio Interestelar, Instituto de Astronomía, Universidad Nacional Autónoma de México, \\ Ciudad Universitaria, 04510 México City, México
}

Received 15 April 2013 / Accepted 11 June 2013

\section{ABSTRACT}

\begin{abstract}
Context. During the transition from the asymptotic giant branch (AGB) to the planetary nebulae phase the circumstellar envelopes of most low- and intermediate-mass stars experience a dramatic change in morphology. CRL 618 exhibits characteristics of both an AGB and post-AGB star. It also displays a spectacular array of bipolar lobes with a dense equatorial region, which makes it an excellent object for studying the development of asymmetries in evolved stars. In recent decades, an elliptical compact HII region located in the center of the nebula has been seen to be increasing in size and flux. This seems to be due to the ionization of the circumstellar envelope by the central star, and it probably indicates the beginning of the planetary nebula phase for CRL 618.

Aims. We aim to determine the physical conditions under which the ionization of the circumstellar envelope of CRL 618 began to take place as well as the subsequent propagation of the ionization front.

Methods. We analyzed interferometric radio continuum data at $\sim 5$ and $22 \mathrm{GHz}$ from observations carried out at seven epochs with the VLA. We traced the flux increase of the ionized region over a period of $\sim 26$ years. We measured the dimensions of the HII region directly from the brightness distribution images to determine the increase of its size over time. For one of the epochs we analyzed observations at six frequencies from which we estimated the electron density distribution. We carried out model calculations of the spectral energy distribution at two different epochs to corroborate our observational results.

Results. We found that the radio continuum flux and the size of the ionized region have been increasing monotonically in the past three decades. The size of the major axis of the HII region shows a dependance on frequency, which has been interpreted as a result of the gradient of the electron density in this direction. The growth of the HII region is due to the expansion of an ionized wind whose mass-loss rate increased continuously for a period of $\sim 100$ years until a few decades ago, when the mass-loss rate experienced a sudden decline. Our results indicate that the circumstellar envelope began to be ionized around 1971, which marks the start of the planetary nebula phase of CRL 618.
\end{abstract}

Key words. stars: late-type - stars: mass-loss - stars: winds, outflows - HII regions

\section{Introduction}

The transition from the asymptotic giant branch (AGB) to the planetary nebula $(\mathrm{PN})$ phase has long been known to be one of the briefest in stellar evolution. As a consequence, the number of Galactic objects in this transitionary stage (the so-called postAGB stars and/or pre-planetary nebulae - PPNe) is small, and our understanding of the physical processes involved in the transition remains limited. It has been observed that during this transition the circumstellar envelopes (CSEs) of the AGB stars experience their most dramatic change in morphology. Therefore, it is important to characterize the physical conditions, as well as the main physical mechanisms, that dominate during the PPN phase to understand the complex shapes and structures that are seen in $\mathrm{PNe}$.

A unique opportunity for studying this rapid transition is provided by the object CRL 618 (Westbrook nebula, GL 618, (R)AFGL 618, IRAS 04395+3601). This source was discovered in the infrared sky survey of Walker \& Price (1975). Westbrook et al. (1975) studied this object thoroughly and suggested for the first time that it might be a nascent planetary nebula. Currently, it is mostly classified as a carbon-rich bipolar PPN that is rapidly evolving toward the PN stage.

The first optical images of this source showed a pair of small nebulosities lying along the east-west direction. The infrared emission was found to be located in the middle of the two visual nebulosities (Westbrook et al. 1975). Gottlieb \& Liller (1976) discovered that the $B$-magnitude of the nebulosities of CRL 618 increased from 18.8 to 16.5 in the period from 1940 to 1975. The first detection of radio continuum toward this source was reported by Wynn-Williams (1977), who detected emission at 5 and $15 \mathrm{GHz}$. Subsequently, Kwok \& Feldman (1981) discovered that CRL 618 was also exhibiting a brightening at radio wavelengths. These authors showed that in the period from 1977 to 1980 the emission at cm wavelengths increased by a factor of 2 . They attributed the rise of the radio flux to the expansion of the ionized region, as a consequence of the increasing temperature of the central star.

High angular resolution images obtained with the Hubble Space Telescope revealed that the morphology of the optical emission of CRL 618 is dominated by a complex of collimated bipolar lobes extending along the east-west direction (Trammell 2000). These structures are composed of shock-excited gas with outflow velocities of up to $200 \mathrm{~km} \mathrm{~s}^{-1}$, inclined by $30-45^{\circ}$ from the plane of the sky (Calvet \& Cohen 1978; Cernicharo et al. 1989; Neri et al. 1992; Cernicharo et al. 2001; SánchezContreras et al. 2002, 2004,; Pardo et al. 2005). In the center of the complex of lobes lies a compact HII region whose ionization is maintained by a B0V star with a temperature of 
$\simeq 27000-32000 \mathrm{~K}$, a mass of $\simeq 0.8 M_{\odot}$, and a luminosity of $\simeq 1-4 \times 10^{4} L_{\odot}($ Westbrook et al. 1975; Calvet \& Cohen 1978; Kaler et al. 1978; Schmidt \& Cohen 1981; Kwok \& Bignell 1984; Goodrich et al. 1991; Knapp et al. 1993). The spectrum of this region is different from that of the lobes, indicating that the gas is completely photoionized with an electronic density $n_{\mathrm{e}} \sim 10^{5}-10^{7} \mathrm{~cm}^{-3}$. The expansion velocity of the gas in this region seems to be $v_{\exp } \sim 30 \mathrm{~km} \mathrm{~s}^{-1}$, much slower than that of the shocked lobes (Sánchez-Contreras et al. 2002).

In this article, we concentrate on the central HII region of CRL 618. Interferometric radio images of this region revealed that the radio emission has a more or less elliptical brightness distribution whose size was found to be about 0 "'.12 $\times 0$ 0".36 at $\sim 22 \mathrm{GHz}$ (Kwok \& Bignell 1984). While the major axis (east-west direction) of the ionized region increases as a function of the observation wavelength, the minor axis (north-south direction) remains constant. This indicates that the HII region is ionization-bound in the north-south direction, suggesting an enhancement of the neutral material in this direction, which is consistent with the presence of a dense molecular torus (Sánchez-Contreras \& Sahai 2004; Sánchez-Contreras et al. 2004). As mentioned above, the radio continuum exhibited a considerable increase of flux since it was detected for the first time until around the end of 1980. Subsequent observations reported that the emission at $\mathrm{cm}$ wavelengths showed no significant increase (Martín-Pintado et al. 1988, 1993, 1995).

The millimeter emission of the compact HII region has also been found to be changing its flux with time (Martín-Pintado et al. 1988; Walmsley et al. 1991; Knapp et al. 1993). However, the exact nature and significance of this rise of the flux remains unclear. Martín-Pintado et al. (1988) suggested that the growth of the mm emission they observed could be explained in terms of an ionized wind whose mass-loss rate has been increasing in the last decades. However, a recent decrease in the mm continuum flux suggests that the mass-loss rate has slowed down in the past few years. These authors modeled the spectral energy distribution and the recombination lines, assuming an $r^{-2}$ power-law for the electron density. From the radio recombination-line emission they derived an electron temperature for the ionized gas of $T_{\mathrm{e}} \sim 13000 \mathrm{~K}$. The value for the electron density in the center of the HII region that fitted their data best was on the order of $10^{7} \mathrm{~cm}^{-3}$, and the expansion velocity of the ionized gas was found to be $\sim 20 \mathrm{~km} \mathrm{~s}^{-1}$.

We present the results of analyzing radio continuum observations spanning more than 25 years. We show that the radio continuum emission has continued its rise since it was discovered, and reveal for the first time the evolution of the expansion of the HII region by directly measuring the change of its size over time. The details of the observations, data calibration, and reduction are presented in Sect. 2 of this paper. The measurements obtained from the data are reported in Sect. 3. In Sect. 4 we derive the physical parameters in the compact HII region and interpret our results. In Sect. 5 we discuss the implications of our results for the nature of CRL 618 and the possible scenarios that could explain our observations. Finally, in Sect. 6 we present the conclusions of this work.

The distance to CRL 618 remains somewhat uncertain, with quoted values varying between 0.9 and $1.8 \mathrm{kpc}$ (Goodrich et al. 1991; Schmidt \& Cohen 1981; Knapp et al. 1993; Sánchez-Contreras et al. 2004). The value that has been used most recently in the literature is that obtained by Goodrich et al. (1991), $D=0.9 \mathrm{kpc}$. We assume this value for the distance of CRL 618 throughout this article, unless stated otherwise.

\section{Observations and results}

To investigate the structural evolution of the structure of the HII region in CRL 618, we have analyzed seven Very Large Array (VLA) observations collected in the years 1982, 1983, 1990, 1992, 1995, 1998, and 2007. All these data sets include observations at the same wavelength $(1.3 \mathrm{~cm})$ and in the same configuration (A) of the array. Therefore they can be directly compared with one another to derive the evolution of the size and flux of the source at this specific wavelength $(v \approx 22 \mathrm{GHz})$. In addition, the data obtained in 1983 include observations at $5 \mathrm{GHz}$ and those obtained in 1998 included observations at six frequency bands. The data calibration and the imaging were made using the AIPS package of the NRAO ${ }^{1}$. After the initial calibration, using extragalactic compact sources as calibrators, we performed phase self-calibration on all the data sets. Subsequently, clean images of the source were obtained using natural weighting (robust parameter $=5$ in the task IMAGR of AIPS). The main parameters of the calibration and the resulting images are given in Tables 2 and 3. The typical size of the synthesized beam at $22 \mathrm{GHz}$ was $\sim 0$.' 1 , thus we adopted this size as a common value for the circular clean beam used during image reconstruction at this frequency of all epochs. The flux density was measured by integrating the emission within a box that contained the whole source. Since the morphology of this source is very simple, we measured its size by fitting a two-dimensional Gaussian model to the emission (we verified that our main results and conclusions are not affected by adopting different fitting models to our data). The results from the fits are shown in Tables 4 and 6. For each observation we followed a somewhat different calibration strategy, therefore we will now describe in some detail the calibration procedures followed for each one.

\subsection{2 observation}

The first observations were carried out in 1982 on June 24 (epoch 1982.48) and were first reported by Kwok \& Bignell (1984) project code: BIGN. The correlator was set up to the continuum mode with a total bandwidth of $50 \mathrm{MHz}$. The observations consisted of one single scan on 3C $48(0134+329$ - used as flux calibrator), 3C 119 (used as phase calibrator), and CRL 618, respectively. The total observation time on the target source was $30 \mathrm{~min}$. The flux scale was determined by assuming a flux density of $1.27 \mathrm{Jy}$ for $3 \mathrm{C} 48$. The bootstrapped flux density of the phase calibrator was $0.97 \pm 0.04 \mathrm{Jy}$. The flux density of CRL 618 was found to be $205 \pm 11 \mathrm{mJy}$. Kwok \& Bignell (1984) did not explicitly quote an integrated flux, but mentioned that the sum of all clean components in their image was $214 \pm 7 \mathrm{mJy}$. Therefore our value well agrees with the previous measurement.

\subsection{3 observation}

The data of the second observation that we analyzed was obtained in 1983 on October 9 (epoch 1983.77) and has not been published before (project AK0094). The observations were made in continuum mode at frequencies 5 and $22 \mathrm{GHz}$ with an effective bandwidth of $100 \mathrm{MHz}$. We calibrated the data at the two frequencies. The main flux-calibrator was 3C 286 $(1328+307)$ and the phase calibrator was J0403+260 for the observations at both frequencies. The total observation time on the

1 The National Radio Astronomy Observatory is a facility of the National Science Foundation operated under cooperative agreement by Associated Universities, Inc. 
Table 1. Flux densities for 3C 84 in the year 1990.

\begin{tabular}{|c|c|c|c|}
\hline Date of observation & $v(\mathrm{GHz})$ & $S_{v}(\mathrm{Jy})$ & Project \\
\hline \multirow[t]{2}{*}{15 February 1990} & 22.2851 & $34.10 \pm 0.55$ & AR416 \\
\hline & 22.2320 & $33.64 \pm 0.70$ & \\
\hline \multirow[t]{2}{*}{17 February 1990} & 22.2851 & $34.38 \pm 0.60$ & AR216 \\
\hline & 22.2320 & $34.71 \pm 0.86$ & \\
\hline \multirow[t]{2}{*}{04 March 1990} & 22.2851 & $36.02 \pm 0.30$ & AR216 \\
\hline & 22.2305 & $36.38 \pm 0.68$ & \\
\hline \multirow[t]{2}{*}{12 April 1990} & 22.4851 & $38.42 \pm 2.25$ & TT001 \\
\hline & 22.4351 & $38.31 \pm 2.24$ & \\
\hline
\end{tabular}

target source at $22 \mathrm{GHz}$ was $\sim 45 \mathrm{~min}$, whereas the observation at $5 \mathrm{GHz}$ lasted only an integration time of 10 seconds. The flux densities of the phase calibrator and the target source were scaled assuming that the flux density of 3C 286 is 7.5 and $2.57 \mathrm{Jy}$ at 5 and $22 \mathrm{GHz}$, respectively. The bootstrapped flux of the source $\mathrm{J} 0403+260$ was $1.15 \pm 0.01 \mathrm{Jy}$, and $0.68 \pm 0.02 \mathrm{Jy}$ at 5 and $22 \mathrm{GHz}$, respectively. For the observation at $22 \mathrm{GHz}$, we performed self-calibration of the data after the initial calibration, using a model image from the previous epoch (1982.48).

\subsection{0 observation}

The third observation was obtained as part of the project AM287 on March 29, 1990 (epoch 1990.24), and was published by Martín-Pintado et al. (1993). The project was designed to investigate the distribution of ammonia in the surroundings of CRL 618, so the data were collected in spectral line mode. The frequency of observation was that of the $\mathrm{NH}_{3}(2,2)$ inversion line at $23.72 \mathrm{GHz}$. In the original data, the quasar $3 \mathrm{C} 84(0316+413)$ was used both as phase- and flux-calibrator (with an assumed flux at $22 \mathrm{GHz}$ of $28 \mathrm{Jy}$ ). However, 3C 84 is now known to be variable. To estimate the flux of 3C 84 at the epoch of the CRL 618 observations, we searched the VLA archive for observing sessions in February, March, and April 1990, in which both 3C 84 and a standard flux calibrator were observed at $\lambda=1.3 \mathrm{~cm}$. Four such sessions were found (Table 1) and were calibrated following standard procedures. The flux of $3 \mathrm{C} 84$ obtained from these data appears to be 34-38 Jy (see Table 1). An independent confirmation for this high flux comes from Teräsranta et al. (1992), who monitored the $22 \mathrm{GHz}$ flux of 3C 84 between January 1986 and June 1990. For the period February-April 1990, they reported fluxes of about $38 \mathrm{Jy}$, in excellent agreement with the values found here from the VLA data. The flux of 3C 84, appropriate for the date of the CRL 618 observations, was estimated by interpolating the last two observations in Table 2 to be $37.7 \mathrm{Jy}$. Note that this is $35 \%$ higher than the flux assumed by MartínPintado et al. (1993). The spectral line data were processed using standard AIPS procedures, including bandpass calibration. The central channels (at $-80 \mathrm{~km} \mathrm{~s}^{-1}<v_{\mathrm{LSR}}<-25 \mathrm{~km} \mathrm{~s}^{-1}$ ) show significant absorption by $\mathrm{NH}_{3}$ (see also Martín-Pintado \& Bachiller 1992; Martín-Pintado et al. 1993, 1995). However, no such absorption is seen at $v_{\mathrm{LSR}}>-20 \mathrm{~km} \mathrm{~s}^{-1}$. Thus, we averaged 17 absorption-free channels at $v_{\mathrm{LSR}}>-20 \mathrm{~km} \mathrm{~s}^{-1}$ to produce a pseudo-continuum data set with an equivalent bandwidth of 6.6 MHz. This is significantly less than the $50 \mathrm{MHz}$ bandwidth of the continuum observations obtained in 1982 (Sect. 2.1). Nevertheless, the noise level achieved is somewhat better in the 1990 than in the 1982 observations because the integration time was significantly longer, $\sim 2.5 \mathrm{~h}$ (Table 2 ). Several iterations of phase self-calibration were applied to the data to improve the quality of the phase calibration. The size of the emitting region is found to be $0^{\prime \prime} .38 \times 00^{\prime \prime} .13$ similar to the values reported by Martín-Pintado et al. (1993): 0".40 × 0'.12. The total flux is found to be $293 \pm 15 \mathrm{mJy}$, significantly higher than the value of $189 \pm 20$ mJy reported by Martín-Pintado et al. (1993). This, of course, mainly reflects the difference in absolute flux calibration related to the updated flux density of $3 \mathrm{C} 84$.

\subsection{2 observation}

The fourth observation was obtained in 1992 on December 22 (epoch 1992.98) and corresponds to the data published by Martín-Pintado et al. (1995) - project code: AM337. Since the primary goal of that observation was the study of the $\mathrm{NH}_{3}(3,3)$ transition, the observations were obtained in spectral mode at the frequency $v=23.872 \mathrm{GHz}$. To produce a continuum image, we averaged 20 line-free channels corresponding to $v_{\mathrm{LSR}} \geq$ $-20 \mathrm{~km} \mathrm{~s}^{-1}$ (the spectra shown by Martín-Pintado \& Bachiller 1992 clearly show that no absorption is present at these velocities). This resulted in a bandwidth of about $4 \mathrm{MHz}$. The observations consisted of scans on CRL 618, interleaved with scans on the calibrators $\mathrm{J} 0359+509$ and 3C 84 for phase-calibration purposes. The total observation time on the target source was $2.75 \mathrm{~h}$. One scan on the calibrator $3 \mathrm{C} 48$ was made at the end of the observations. Martín-Pintado et al. (1995) used 3C 84 as amplitude calibrator assuming a flux at $1.3 \mathrm{~cm}$ of $28 \mathrm{Jy}$. The calibrator 3C 84 is known to have a structure that departs significantly from a point source at $v=22 \mathrm{GHz}$. In addition, as mentioned above, this calibrator is also known to be variable. Because of these reasons, we calibrated the amplitudes instead using the calibrator $3 \mathrm{C} 48$, for which the flux density was assumed to be $1.2 \mathrm{Jy}$. We note that the bootstrapped flux of $3 \mathrm{C} 84$ obtained using 3C 48 as primary flux calibrator is $26.6 \pm 0.5 \mathrm{Jy}$, which is sightly lower than the value assumed by Martín-Pintado (1995). The bootstrapped flux of $0355+508$ was $3.1 \mathrm{Jy}$. After a few self-calibration iterations, we obtained an image of CRL 618 from which we obtained its parameters. The integrated flux is $365 \pm 9 \mathrm{mJy}$, which is significantly higher than the value reported by Martín-Pintado et al. (1995; $250 \pm 20 \mathrm{mJy}$; even though they assumed a higher value for the flux of $3 \mathrm{C} 84$ than the bootstrapped value obtained from our calibration). This discrepancy could be mainly due to the difference of the amplitudecalibration procedures that we followed. However, our value is consistent with the monotonic increasing trend that the flux has followed during the past decades, which is also seen from the data at $5 \mathrm{GHz}$. This indicates that the value obtained by MartínPintado et al. (1995) was affected in the calibration process.

\subsection{5 observation}

The fifth observation was obtained in 1995 on August 3 (epoch 1995.59) as part of the project AM0486. These data have not been published before. The observations were made in spectral line mode with a bandwidth of $\sim 6 \mathrm{MHz}$ centered at the frequency $v=22.693 \mathrm{GHz}$. Since we did not find line emission across this bandwidth, we averaged the default channel range (i.e., the center $75 \%$ ) to produce the continuum data set. The primary flux and phase calibrators were 3C 286 and 3C 84, respectively. The assumed flux density for the primary flux calibrator was $2.55 \mathrm{Jy}$ and the bootstrapped flux for the phase calibrator was $19.4 \pm 0.5 \mathrm{Jy}$. The total observation time on the target source was $\sim 1.2 \mathrm{~h}$. The data were initially calibrated using the quasars, and subsequently the target source was self-calibrated in phase. 
Table 2. Calibration parameters of the observations at $22 \mathrm{GHz}$.

\begin{tabular}{|c|c|c|c|c|c|c|}
\hline Date of observation & Flux calibrator & $S_{v}{ }^{1}$ & Phase calibrator & $S_{v}^{2}$ & rms noise $^{3}$ & Beam $^{4}$ \\
\hline (year) & (name) & (Jy) & (name) & (Jy) & $\left(\mathrm{Jy} \mathrm{beam}^{-1}\right)$ & $\left({ }^{\prime \prime},{ }^{\circ}\right)$ \\
\hline 24 June 1982 & $3 \mathrm{C} 48$ & 1.27 & $3 \mathrm{C} 119$ & 0.97 & $7.0 \times 10^{-4}$ & $0.11 \times 0.09,-57$ \\
\hline 9 October 1983 & $3 \mathrm{C} 286$ & 2.57 & $\mathrm{~J} 0403+260$. & 0.68 & $1.2 \times 10^{-3}$ & $0.10 \times 0.08,-71$ \\
\hline 29 March 1990 & $3 \mathrm{C} 84$. & 37.7 & $3 \mathrm{C} 84$. & 37.7 & $6.5 \times 10^{-4}$ & $0.13 \times 0.09,-69$ \\
\hline \multirow[t]{2}{*}{22 December $1992 \ldots$} & $3 \mathrm{C} 48$ & 1.2 & $3 \mathrm{C} 84$. & 26.6 & $5.6 \times 10^{-4}$ & $0.09 \times 0.08,-42$ \\
\hline & & & $\mathrm{J} 0359+509$ & 3.1 & $5.6 \times 10^{-4}$ & $0.09 \times 0.08,-42$ \\
\hline 3 August 1995 & $3 C 286$ & 2.55 & $3 \mathrm{C} 84 \ldots \ldots$ & 19.4 & $1.0 \times 10^{-3}$ & $0.11 \times 0.10,-28$ \\
\hline 2 May 1998 & $\mathrm{~J} 0443+3441$ & 0.35 & $\mathrm{~J} 0443+3441 \ldots$ & 0.35 & $4.0 \times 10^{-4}$ & $0.14 \times 0.10,-79$ \\
\hline 9 July 2007 & $3 \mathrm{C} 48 \ldots \ldots$ & 1.27 & $\mathrm{~J} 04183+38015$ & 5.2 & $1.3 \times 10^{-4}$ & $0.12 \times 0.09,-81$ \\
\hline
\end{tabular}

Notes. ${ }^{(1)}$ Assumed value for the primary flux calibrator. ${ }^{(2)}$ Bootstrapped flux obtained using the AIPS task GETJY. ${ }^{(3)}$ rms noise in the image of the target source made with natural-robust weighting. ${ }^{(4)}$ Size and position angle of the synthesized beam using natural-robust weighting.

Table 3. Calibration parameters of the observation on epoch 1998.33.

\begin{tabular}{ccccc}
\hline \hline Frequency & Flux/phase calibrator & $S_{V}{ }^{1}$ & rms noise $^{3}$ & Beam $^{4}$ \\
\hline$(\mathrm{GHz})$ & (name) & $(\mathrm{Jy})$ & $\left(\mathrm{Jy} \mathrm{beam}^{-1}\right)$ & $\left({ }^{\prime \prime},{ }^{\circ}\right)$ \\
\hline 1.43 & $\mathrm{~J} 0443+3441$ & 0.70 & $5.3 \times 10^{-5}$ & $2.0 \times 1.6,-66$ \\
4.89 & $\mathrm{~J} 0443+3441$ & 0.93 & $3.5 \times 10^{-5}$ & $0.63 \times 0.52,-72$ \\
8.44 & $\mathrm{~J} 0443+3441$ & 0.74 & $4.7 \times 10^{-5}$ & $0.34 \times 0.28,-71$ \\
14.96 & $\mathrm{~J} 0443+3441$ & 0.50 & $1.7 \times 10^{-4}$ & $0.20 \times 0.16,-77$ \\
22.49 & $\mathrm{~J} 0443+3441$ & $0.35^{2}$ & $3.9 \times 10^{-4}$ & $0.14 \times 0.10,-79$ \\
43.31 & $\mathrm{~J} 0443+3441$ & 0.20 & $3.1 \times 10^{-4}$ & $0.046 \times 0.043,30$ \\
\hline
\end{tabular}

Notes. ${ }^{(1)}$ Assumed value for the primary flux calibrator, obtained from the NRAO VLA calibrators webpage. ${ }^{(2)}$ Interpolated value from the SED of the flux calibrator. ${ }^{(3)}$ rms noise in the image of the target source made with natural-robust weighting. ${ }^{(4)}$ Size and position angle of the synthesized beam using natural-robust weighting.

\subsection{8 observation}

The data corresponding to the sixth observation were obtained in 1998 on May 2 (epoch 1998.33) as part of the project AW048. These data have not been published before. The observations were made in continuum mode with a total bandwidth of $100 \mathrm{MHz}$. The array was split into two subarrays: VLA-A1 and VLA-A2, with 12 and 15 antennas, respectively. The subarray A1 included the antennas at the tips of the arms of the array, therefore it contained the longest baselines. Observations of CRL 618 at $v=8$ and $43 \mathrm{GHz}$ were made using the subarray A1, whereas the subarray A2 was used to carry out observations at $v=1.4,5,15$ and $22 \mathrm{GHz}$. We calibrated the data at the six different frequencies. The details of the calibration at the different frequencies are shown in Table 3. After the initial calibration, all data corresponding to CRL 618 were self-calibrated in phase.

\subsection{7 observation}

The data from the last observation that we analyzed was obtained in 2007 on July 9 (epoch 2007.52) in continuum mode with $100 \mathrm{MHz}$ of equivalent bandwidth (project code: AL698). These observations were carried out using the fast-switching technique, which consists of rapidly alternating observations of the source and the phase calibrator $(\mathrm{J} 0418+380)$ with a cycle time of $2 \mathrm{~min}$. The total observation time on the target source was $\sim 35 \mathrm{~min}$. The primary flux calibrator was 3C 48, whose flux was assumed to be $1.27 \mathrm{Jy}$. The bootstrapped flux of the phase calibrator was $5.2 \pm 0.1 \mathrm{Jy}$. At the time of the observations, about half of the VLA antennas had already been upgraded to EVLA status.

\section{Evolution of the radio continuum of CRL 618}

Observations over the past decades have revealed that the emission of CRL 618 at $\mathrm{mm}$ and $\mathrm{cm}$ wavelengths exhibits changes over time (Kwok \& Feldman 1981; Kwok \& Bignell 1984; Martín-Pintado et al. 1995; Knapp et al. 1995; Sánchez-Contreras et al. 2004 and references therein). Particularly, at $\mathrm{cm}$ wavelengths the emission shows a more or less monotonic increase trend. Because the spectral index $\alpha$, defined as $S_{v} \propto v^{\alpha}$, of the radio continuum emission is very close to the value +2 , the increase of the flux has been explained in terms of an expanding optically thick ionized region. Knapp et al. (1995) presented a plot showing the increase of the radio continuum flux at $\sim 5 \mathrm{GHz}$ using data collected from 1974 until 1991; they extrapolated the value of the time that corresponds to flux equal to zero and estimated that the ionization of the source began in about 1965.

From our analysis we have obtained measurements of the flux density of CR 618 at $22 \mathrm{GHz}$ at seven epochs ranging from 1982 until 2007 (see Sect. 2). Additionally, we obtained the values of the flux at $5 \mathrm{GHz}$ in two epochs to which we added the values of seven more epochs collected from the literature. The results are presented in Tables 4 and 5; in Fig. 1 we have plotted the value of the flux at $\sim 22$ and $\sim 5 \mathrm{GHz}$ as a function of time, respectively. Since the frequency of the observation was not exactly the same for all epochs (see Table 4), we scaled the values of the flux to a common frequency $(22.485$ and $4.885 \mathrm{GHz}$, respectively) for all epochs, assuming that the spectral index has not changed considerably (see Sect. 4). From this figure we see that the flux at both frequencies has increased by a factor of more than two since the first observations. The rise of the flux has been roughly linear with time, but to also account for the growth of the 
D. Tafoya et al.: Rapid angular expansion of the ionized core of CRL 618
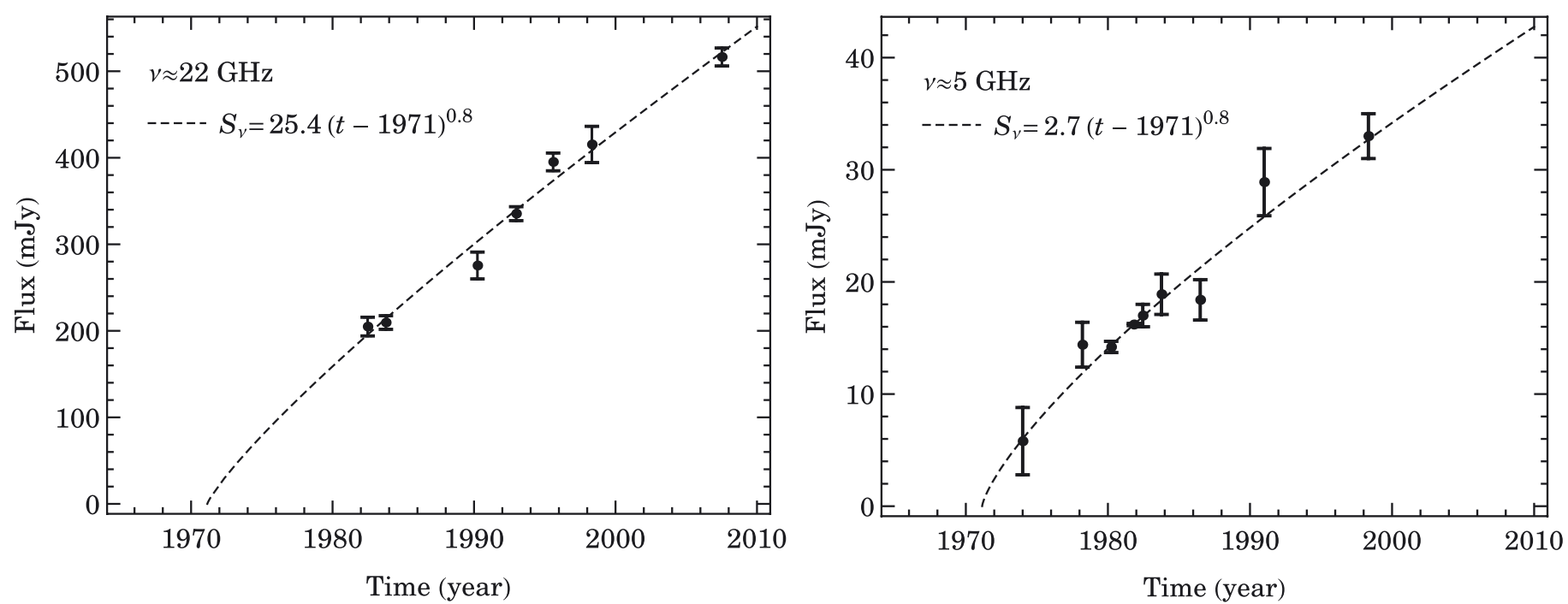

Fig. 1. Increase of the CRL 618 flux density at $22 \mathrm{GHz}$ (left panel) and $5 \mathrm{GHz}$ (right panel) as a function of time. The dashed line is the result of a simultaneous power-law fit to the data of the flux at $22 \mathrm{GHz}$ and $5 \mathrm{GHz}$, and the size of the source at $22 \mathrm{GHz}$ (Fig. 2). The numerical expressions of the fits are also shown in the panels. We set the starting time of the ionization as a common parameter of the fit (see main text).

Table 4. Parameters of CRL 618 at $\sim 22 \mathrm{GHz}$.

\begin{tabular}{cccccc}
\hline \hline Epoch & Frequency & \multicolumn{1}{c}{$S_{v}$} & Minor axis & Major axis & PA \\
\hline year & \multicolumn{1}{c}{ GHz } & \multicolumn{1}{c}{ mJy } & arcsec & arcsec & degrees \\
\hline 1982.48 & 22.485 & $205 \pm 11$ & $0.103 \pm 0.009$ & $0.333 \pm 0.015$ & $83 \pm 1$ \\
1983.77 & 22.485 & $210 \pm 8$ & $0.115 \pm 0.012$ & $0.302 \pm 0.019$ & $88 \pm 2$ \\
1990.24 & 23.722 & $300 \pm 16$ & $0.133 \pm 0.006$ & $0.383 \pm 0.013$ & $81 \pm 1$ \\
1992.98 & 23.872 & $368 \pm 8$ & $0.146 \pm 0.004$ & $0.414 \pm 0.009$ & $82 \pm 1$ \\
1995.59 & 22.693 & $401 \pm 10$ & $0.150 \pm 0.010$ & $0.458 \pm 0.022$ & $82 \pm 1$ \\
1998.33 & 22.485 & $415 \pm 21$ & $0.161 \pm 0.007$ & $0.455 \pm 0.014$ & $82 \pm 1$ \\
2007.52 & 22.485 & $517 \pm 10$ & $0.185 \pm 0.001$ & $0.499 \pm 0.002$ & $83 \pm 1$ \\
\hline
\end{tabular}

Table 5. Flux of CRL 618 at $\sim 5 \mathrm{GHz}$.

\begin{tabular}{cccc}
\hline \hline Epoch & Frequency & $S_{v}$ & Telescope \\
\hline year & $\mathrm{GHz}$ & $\mathrm{mJy}$ & \\
\hline 1974.0 & 5.00 & $6 \pm 3$ & Cambridge 5-km \\
1978.2 & 4.80 & $14 \pm 2$ & Effelsberg 100m $^{2}$ \\
1980.2 & 4.89 & $14.2 \pm 0.5$ & $\ldots$. NRAO-VLA \\
1981.8 & 4.89 & $16.2 \pm 0.1$ & $\ldots$ NRAO-VLA $^{4}$ \\
1982.5 & 4.89 & $17 \pm 1$ & $\ldots$. NRAO-VLA \\
1983.8 & 4.89 & $18.9 \pm 1.8$ & $\ldots$. NRAO-VLA \\
1986.5 & 4.89 & $18.4 \pm 1.8$ & $\ldots$. NRAO-VLA \\
1991.0 & 4.89 & $26 \pm 3$ & . NRAO-VLA $^{8}$ \\
1998.3 & 4.89 & $33 \pm 2$ & $\ldots$ NRAO-VLA $^{6}$ \\
\hline
\end{tabular}

References. ${ }^{(1)}$ Wynn-Williams (1977); ${ }^{(2)}$ Mross et al. (1981); ${ }^{(3)}$ Kwok \& Feldman (1981); ${ }^{(4)}$ Spergel et al. (1983); ${ }^{(5)}$ Kwok \& Bignell (1984); (6) This work; ${ }^{(7)}$ Zijlstra et al. (1989); ${ }^{(8)}$ Knapp et al. (1995).

size as a function of time, we performed a simultaneous powerlaw fit (see below) in which we set the ionization starting time as a common parameter.

Even though images of this source at radio frequencies have been obtained and presented in previous works, so far the change of the size has been inferred only from the increase of the flux, and assuming that the entire emission is optically thick (Kwok \& Feldman 1981; Spergel et al. 1983; Knapp et al. 1995). In this work we characterize the evolution of the size of ionized region by directly measuring the change of the brightness distribution from the images. Because the morphology of the source is not

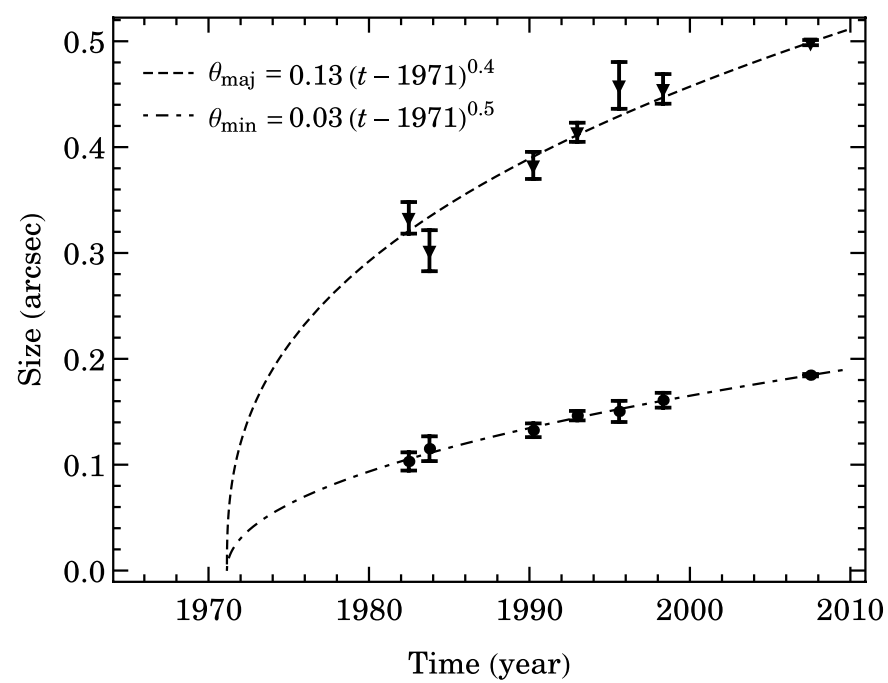

Fig. 2. Increase of the size of CRL 618 at $22 \mathrm{GHz}$ as a function of time. The dashed and dot-dashed lines are the fits to the major and minor axes, respectively. The fit was obtained by performing a simultaneous power-law fit to the data of the flux at $22 \mathrm{GHz}$ and $5 \mathrm{GHz}$ (see Fig. 1), and the size of the source at $22 \mathrm{GHz}$. The numerical expressions of the fits are also shown. We set the ionization starting time as a common parameter of the fit (see main text).

too complex at radio wavelengths, we measured its size by fitting an elliptical Gaussian model to its brightness distribution. From the fit we obtained the values of the major and minor axes and the position angle of the major axis; the results are presented in Table 4. In Fig. 2 we plot the increase with time of the size of the ionized region of CRL 618 at $v=22 \mathrm{GHz}$ using the data from our elliptical Gaussian fit to the images; the inverted triangles correspond to the major axis, whereas the circles correspond to the minor axis of the source. In the same fashion as the flux, the size of the source has been experiencing a monotonic increase. We note that the major axis of the epoch 1983.77 is slightly smaller than that of the previous epoch, but the noise level of the visibilities phases of the epoch 1983.77 was significantly higher, resulting in a larger uncertainty in the size determination. A linear fit to the size as a function of time yields a zero-flux-time that 

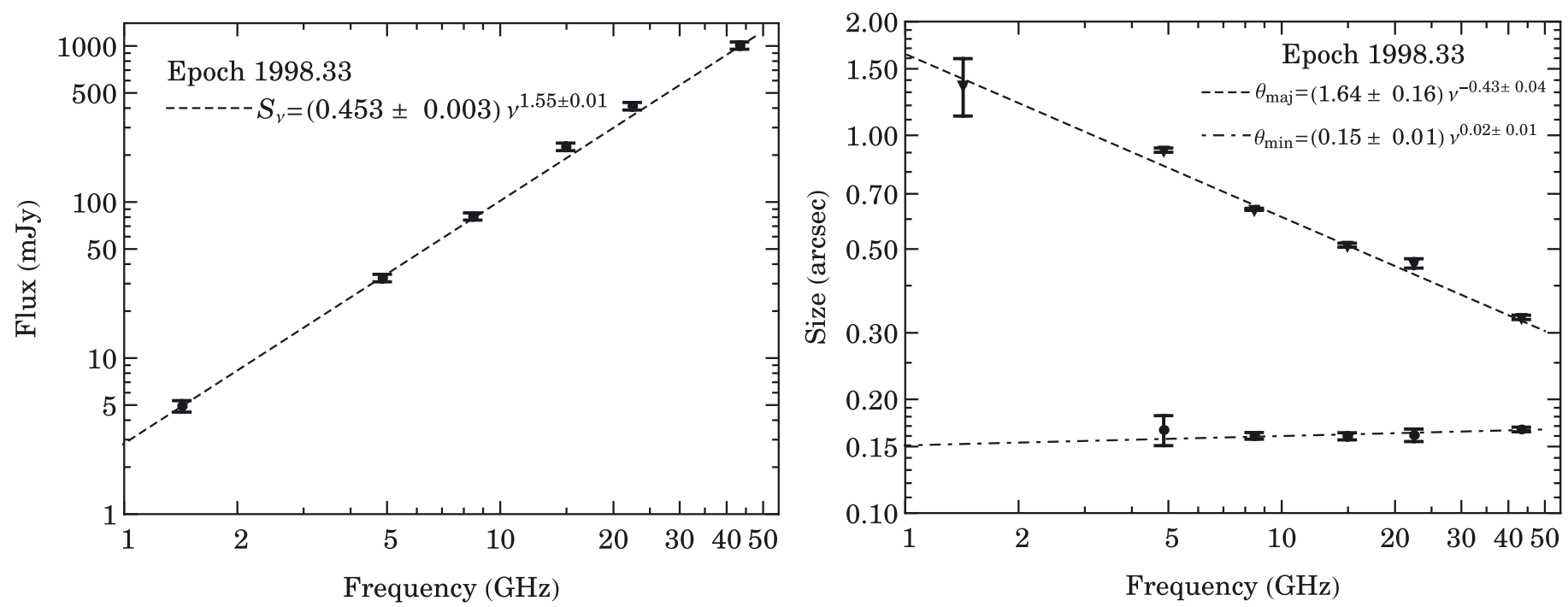

Fig. 3. Dependence of radio flux and size of CRL 618 with Frequency. The data correspond to the observations carried out in the epoch 1998.33. The minor axis of the source at $v=1.46 \mathrm{GHz}$ was not resolved, thus we only show the value of the major axis. The dashed and dot-dashed lines represent least squares fits to the data, assuming a power-law model. The numerical expressions of the fits are also shown in the panels.

corresponds to the beginning of the ionization, which differs by more than 30 years from to that obtained by performing the same type of fit to the flux data shown in Fig. 1. This discrepancy is significant, even considering the errors of the fit. Because of this, we conclude that neither the flux nor the size have followed a linear increase with time. Thus, to a first approximation, we fitted a power-law of the form $a\left(t-t_{0}\right)^{b}$ to all data sets, setting as a common parameter the ionization starting time, $t_{0}$. The expressions of the fitted power-laws for the flux, major and minor axes, are the following:

$$
\begin{aligned}
& {\left[\frac{S_{22 \mathrm{GHz}}}{\mathrm{mJy}}\right]=(25.4 \pm 10) \times\left(\left[\frac{t}{\text { year }}\right]-(1971 \pm 2)\right)^{0.8 \pm 0.1},} \\
& {\left[\frac{S_{5 \mathrm{GHz}}}{\mathrm{mJy}}\right]=(2.7 \pm 1.3) \times\left(\left[\frac{t}{\text { year }}\right]-(1971 \pm 2)\right)^{0.8 \pm 0.1},} \\
& {\left[\frac{\theta_{\text {maj }}}{\operatorname{arcsec}}\right]=(0.13 \pm 0.02) \times\left(\left[\frac{t}{\text { year }}\right]-(1971 \pm 2)\right)^{0.4 \pm 0.1},} \\
& {\left[\frac{\theta_{\text {min }}}{\operatorname{arcsec}}\right]=(0.03 \pm 0.01) \times\left(\left[\frac{t}{\text { year }}\right]-(1971 \pm 2)\right)^{0.5 \pm 0.1} .}
\end{aligned}
$$

From these results we notice that the increase of the flux is proportional to the increase of the source area, i.e., the exponent of the power-law fit of the flux is, within the errors, the sum of the exponents of the power-law fits of the minor and major axes. This result indicates that indeed the rise of the radio continuum is mainly due to the expanding projected area of the optically thick ionized region. The expansion rate of the major axis is higher than that of the minor axis by a factor of nearly two, currently it

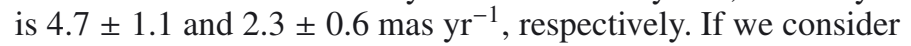
a distance to the source $D=900 \mathrm{pc}$ and an inclination of the major axis with respect to the plane of the sky of $\sim 25^{\circ}$ (SánchezContreras et al. 2002), the measured expansion rates translate into deprojected linear velocities of $22 \pm 5$ and $10 \pm 2 \mathrm{~km} \mathrm{~s}^{-1}$ for the major and minor axes, respectively. The fit gives a year for the beginning of the ionization about 1971, which is more recent that previously considered in other works.
Table 6. Parameters of CRL 618 for epoch 1998.33

\begin{tabular}{ccccc}
\hline \hline Line frequency & $S_{v}$ & Minor axis & Major axis & PA \\
\hline $\mathrm{GHz}$ & $\mathrm{mJy}$ & $\operatorname{arcsec}$ & $\operatorname{arcsec}$ & degrees \\
\hline 1.46 & $5 \pm 1$ & $\ldots \ldots \ldots \ldots$ & $1.360 \pm 0.236$ & $89 \pm 3$ \\
4.89 & $33 \pm 2$ & $0.166 \pm 0.015$ & $0.914 \pm 0.012$ & $87 \pm 1$ \\
8.44 & $81 \pm 4$ & $0.160 \pm 0.003$ & $0.637 \pm 0.004$ & $82 \pm 1$ \\
14.96 & $226 \pm 13$ & $0.160 \pm 0.003$ & $0.512 \pm 0.006$ & $82 \pm 1$ \\
22.49 & $415 \pm 21$ & $0.161 \pm 0.006$ & $0.458 \pm 0.013$ & $82 \pm 1$ \\
43.31 & $1007 \pm 54$ & $0.166 \pm 0.002$ & $0.330 \pm 0.004$ & $87 \pm 1$ \\
\hline
\end{tabular}

Notes. ${ }^{(1)}$ The minor axis was not resolved at this frequency.

\section{Physical parameters of the ionized region}

In previous works the spectral index of the radio continuum emission at $\mathrm{cm}$ wavelengths of CRL 618 has been considered to be $\alpha=+2$, or very close to this value (e.g. Kwok \& Bignell 1984). This implies that the emitting ionized region has a uniform electron density distribution. Given that the flux of this source evolves with time, one cannot directly compare the flux measurements of one epoch with another. Thus, so far the only reliable spectral index value of the radio emission of this source is the one derived from the measurements at three wavelengths obtained in quasi-simultaneous observations by Kwok \& Bignell (1984). Martín-Pintado et al. (1988) performed a fit to the spectral energy distribution from $\mathrm{cm}$ wavelengths to $\mathrm{mm}$ wavelengths. These authors concluded that the emission could not correspond to an HII region with uniform electron density. They suggested that the electron density must be following a power-law distribution of the form $n_{\mathrm{e}}=A r^{-2}$, which results in a spectral index $\alpha<+2$. Indeed, a power-law fit to the data of Kwok \& Bignell (1984) gives a spectral index $\alpha=+1.71 \pm 0.01$.

Among the data we analyzed in this work are simultaneous observations of the radio continuum of CRL 618 at six different frequencies (see Sect. 2). We measured the flux density of the source at each frequency. Additionally, for each frequency we fitted an elliptical Gaussian model to the brightness distribution to measure the major and minor axes as well as the position angle of the major axis of the emitting region; the results are presented in Table 6. In Fig. 3 we show the integrated flux and the 


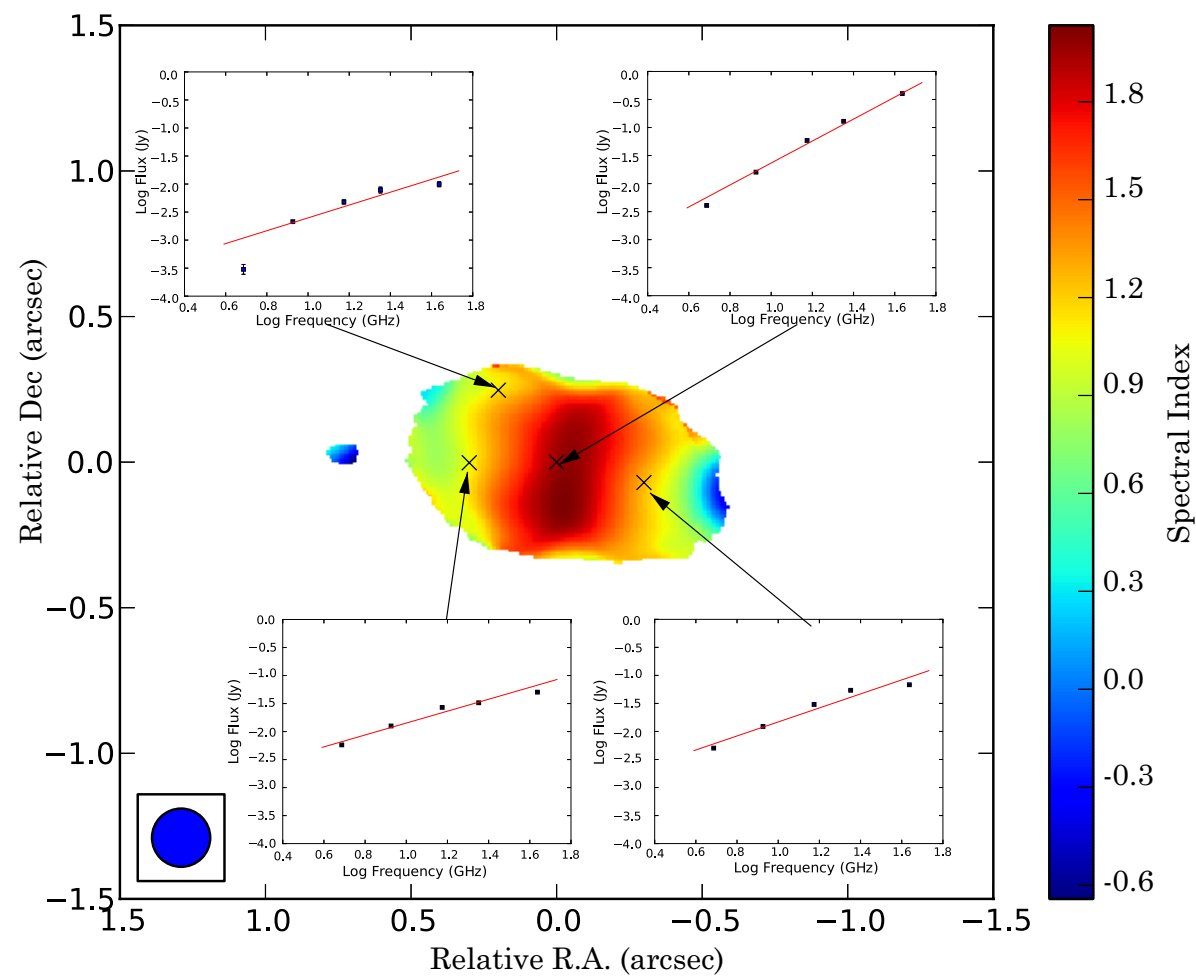

Fig. 4. Spectral index map of CRL 618 made using the emission at five frequencies from 5 to $43 \mathrm{GHz}$. The inner panels show the SED fit for specific points of the source. A common synthesized beam of $0.2 \times 00^{\prime} 2$ was used to make the map.

major and minor axes of CRL 618 as a function of the frequency. A power-law fit to the data of the flux yields a spectral index in the frequency range from 1 to $43 \mathrm{GHz}$ of $\alpha=+1.55 \pm 0.01$. This value is lower than the value derived from the data obtained by Kwok \& Bignell (1984), which implies that the spectral index has been decreasing over time. However, this spectral index results from averaging the emission over the whole source. To obtain more information about the distribution of the ionized gas (i.e., the electron density) we combined the emission at five different frequencies, $5,8.4,14,22$, and $43 \mathrm{GHz}$, to map the spectral index across the source; the result is shown in Fig. 4. In this figure we see that the spectral index of the emission ranges from values near zero to values up to +2 for different parts of the source. The highest values correspond to a band that crosses the source approximately in the north-south direction, and it exhibits a steep gradient in the east-west direction.

For the case in which the depth and electron temperature of the ionized region are constant across the source (below we show that this assumption is a good approximation), this map basically traces the electron density of the plasma. Thus, this spectral map shows that the electron density reaches a maximum on a band in the central region and decreases outward in the eastwest direction, which is the direction of the bipolar lobes seen at optical wavelengths. This electron density gradient is also revealed by the dependance of the major axis on the frequency, which is plotted in the right panel of Fig. 3. From this plot we can see that while the minor axis remains basically constant for all frequencies, the major axis shows a clear dependance on the frequency, $\theta_{\text {maj }} \propto v^{-0.43 \pm 0.04}$. This behavior of the major axis can be explained in terms of the emission becoming optically thin at different distances from the star. When this occurs, the emission brightness decreases rapidly as a function of the optical depth. Reynolds (1986) demonstrated that for a partially optically thick ionized outflow whose width, depth, and electron temperature are constant, the total integrated flux is proportional to the product of the size of the optically thick region and the frequency squared: $S_{v} \propto \theta_{\text {thick }} \cdot v^{2}$. Since we found from our observations (right panel of Fig. 3) that $\theta_{\text {maj }} \propto v^{-0.43}$, assuming that the size of the major axis is basically the size of the optically thick region, we have that $S_{v} \propto v^{2-0.43}=v^{1.57}$, which is exactly the behavior of the total integrated flux that we see in the plot on the left panel of Fig. 3. Therefore, we can consider that the width, depth, and electron temperature of the ionized region are basically constant and that $\theta_{\text {maj }}$ corresponds to the size at which the emission becomes optically thin, $\tau_{v} \approx 1$.

The optical depth along one line-of-sight path of a region of ionized gas can be approximated by the following expression:

$\tau_{v}=8.235 \times 10^{-2}\left[\frac{E M}{\mathrm{~cm}^{-6} \mathrm{pc}}\right]\left[\frac{T_{\mathrm{e}}}{\mathrm{K}}\right]^{-1.35}\left[\frac{v}{\mathrm{GHz}}\right]^{-2.1}$,

where $T_{\mathrm{e}}$ is the electron temperature and $E M$ is the emission measure, defined as $E M=\int n_{\mathrm{e}}^{2} \mathrm{~d} L$, where $n_{\mathrm{e}}$ is the electron density and $\mathrm{d} L$ is the differential path length (Altenhoff et al. 1960). If we assume that the electron density remains more or less constant along one line-of-sight path, we can write the emission measure as $E M=n_{\mathrm{e}}^{2} L$, where $L$ is the total depth of the source in the line-of-sight path. Thus, for a given frequency, from the expression above we can calculate the minimum electron density necessary for the gas to become optically thick along one line-of-sight path as follows.

Assume that the density can be expressed as a power-law of the distance to the star of the form $n_{\mathrm{e}}=A r^{q_{\mathrm{n}}}$. Consider the relation between the major axis and the frequency we obtained from the fit shown in Fig. $6, r_{\tau=1}=0.82 v^{-0.4}$, where we used $r_{\tau=1}=\theta_{\text {maj }} / 2$. Solving for $n_{\mathrm{e}}$ in (1) and assuming that $L$ and $T_{\mathrm{e}}$ do not depend on the distance from the central star or on the observation frequency, we can obtain the following relation:

$A \cdot\left(0.82 v^{-0.4}\right)^{q_{\mathrm{n}}}=3.48\left(T_{\mathrm{e}}^{1.35} v^{2.1} L^{-1}\right)^{1 / 2}$. 


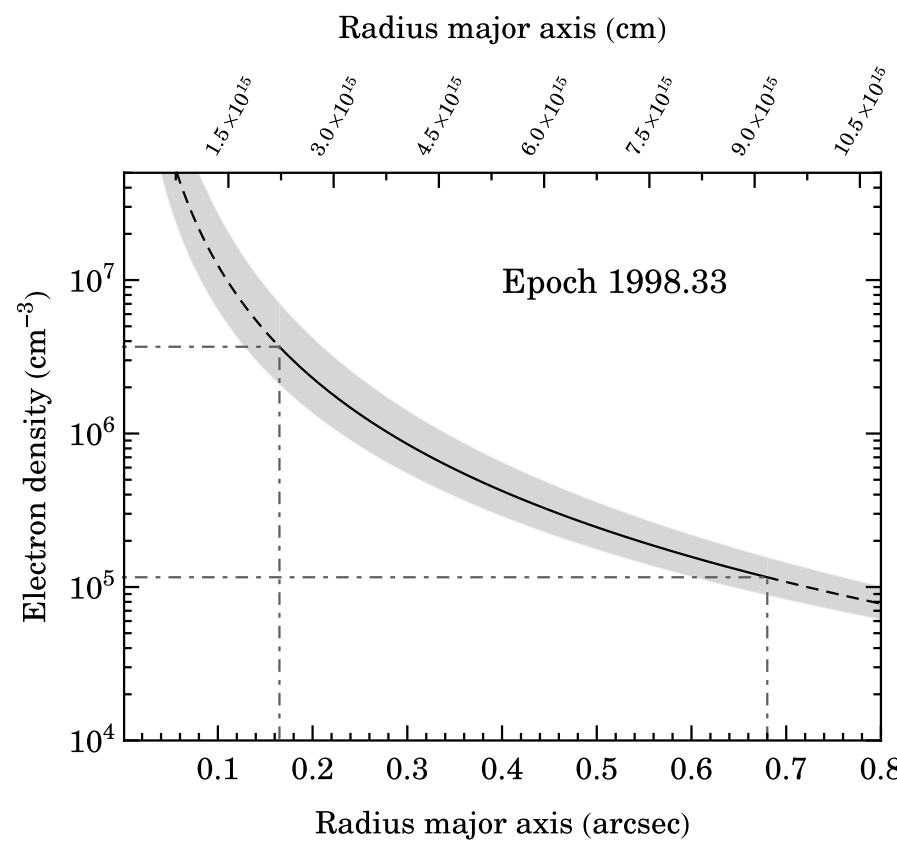

Fig. 5. Dependence of the electron density on the radius for the compact HII region of CRL 618. The solid line indicates the region for which we have measurements of the size of the major axis as a function of frequency (see Fig. 4). The dashed lines are extrapolations of the fit following the same power-law dependance. The gray region represents the uncertainty region due to the error of the fitted parameters. The horizontal/vertical dot-dashed lines indicate the extreme values of the electron density and size of the source, corresponding to the observing frequencies: $v=43.31$ and $1.46 \mathrm{GHz}$ (see Table 6).

By equalizing the frequency exponent on both sides of the equation, we find that $q_{\mathrm{n}}=-2.4 \pm 0.2$. Thus, the expression for the coefficient $A$ is

$A=2.3\left(T_{\mathrm{e}}^{1.35} L^{-1}\right)^{1 / 2}$.

We can furthermore assume that the depth of the region is equal to the width of the minor axis, i.e., $L=\theta_{\min }$, and that the electron temperature is $T_{\mathrm{e}}=13000 \mathrm{~K}$ (Martín-Pintado et al. 1988). Using these values to calculate $A$ and using the value of $q_{\mathrm{n}}$ obtained above, we can write the expression of the electron density along the direction of the major axis as

$\left[\frac{n_{\mathrm{e}, \tau=1}}{\mathrm{~cm}^{-3}}\right]=(4.9 \pm 0.9) \times 10^{4}\left[\frac{r^{-2.4 \pm 0.2}}{\operatorname{arcsec}}\right]$,

where we assumed a distance to the source $D=900$ pc. In Fig. 5 we show a plot of the electron density along the major axis as a function of radius. The gray region corresponds to the estimation errors of the parameters $A$ and $q_{\mathrm{n}}$. This plot shows that the electron density ranges from $n_{\mathrm{e}} \sim 10^{5} \mathrm{~cm}^{-3}$ to more than $n_{\mathrm{e}} \sim 10^{6} \mathrm{~cm}^{-3}$ across the source, with the highest value corresponding to the electron density in the equatorial band seen in Fig. 4. Indeed, the electron density value in this region represents just a lower limit because the emission is optically thick. The solid line in Fig. 5 indicates the range of size and density within which we have observational data (delimited by the dotdashed lines). The dashed lines are extrapolations of the electron density for other values of the radius. Following the same analysis, but using the data obtained by Kwok \& Bignell (1984), we estimated the corresponding parameter values of the power-law of the electron density for that observation epoch. We found a very similar exponent of the power-law, $q_{\mathrm{n}}=-2.4$, but a smaller

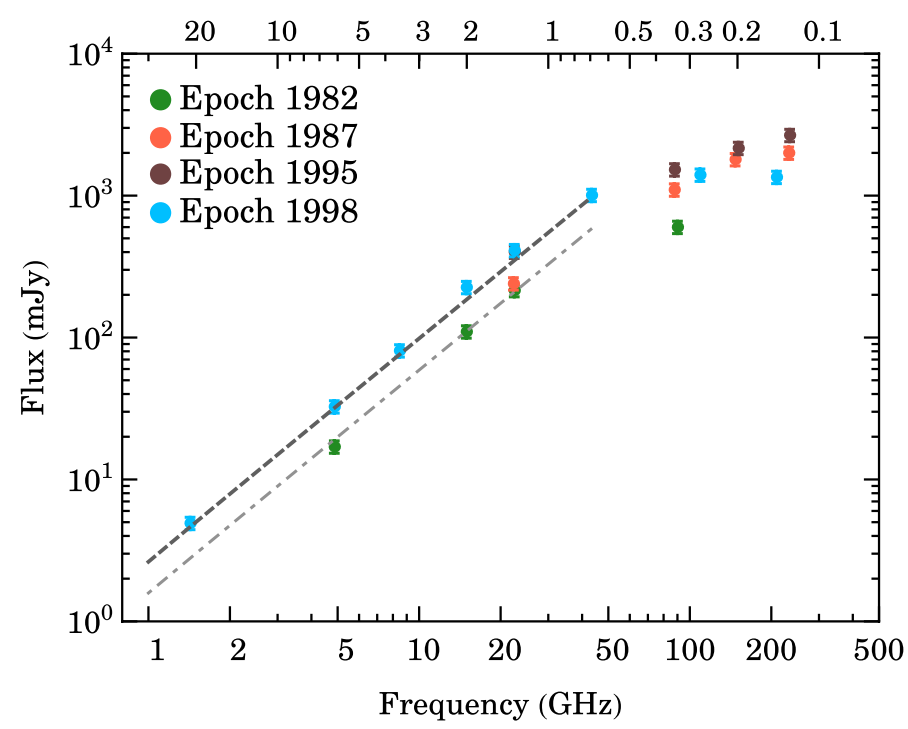

Fig. 6. Spectral energy distribution of the radio continuum emission of CRL 618. The colors indicate the data from the same epoch, as is shown in the upper left corner of the figure. The dashed line corresponds to model calculations for the epoch 1998 (see main text). The dot-dashed line corresponds to model calculations for the epoch 1982 (see main text). The data at wavelengths $<5 \mathrm{~mm}$ were taken from Kwok \& Feldman (1981; extrapolation to epoch 1982); MartínPintado et al. (1988; epoch 1987), Reuter et al. (1997; epoch 1995); Sánchez-Contreras \& Sahai (2004; extrapolation to epoch 1998).

coefficient $A=(4.2 \pm 0.1) \times 10^{4}$, suggesting a lower value for the electron density in the past (see below).

On the other hand, the size of the minor axis does not exhibit a dependance on the frequency. This indicates that the emission is optically thick at all observed frequencies in this direction, which can be directly seen in Fig. 4. From Fig. 5 we see that the electron density in this region must be higher than $\sim 3 \times 10^{6} \mathrm{~cm}^{-3}$. Observations of the molecular emission toward CRL 618 have revealed a dense torus-like structure that extends perpendicular to the bipolar lobes (Sánchez-Contreras \& Sahai 2004). The density of the inner radius of the torus has been estimated to be of the order of $10^{6} \mathrm{~cm}^{-3}$, which perfectly agrees with our results. Since the HII region is ionization-bound in the direction of the minor axis, the increase of its size in this direction is mainly due to the propagating ionization front. Our observations can be successfully modeled by analytical calculations of the expansion rate for an HII region, however, we will present these results somewhere else.

To corroborate that the electron density has been increasing with time in the past, we calculated the spectral energy distribution at two different epochs using a model similar to that of Martín-Pintado et al. (1988). In our calculations, we used the electron density profile given in Eq. (2) (epoch 1998.33) and the profile obtained from the parameters derived from the observations of Kwok \& Bignell (1984; epoch 1982.48). The geometry of the emitting region was assumed to be cylindrical, with the cylinder diameter equal to the measured minor axis. We assumed an electron temperature $T_{\mathrm{e}}=13000 \mathrm{~K}$ and a distance to the source $D=900 \mathrm{pc}$. The calculation results are shown in Fig. 6 as dashed and dot-dashed lines. The data from the different epochs are color-coded. The calculations fit the data very well. This result confirms that the assumptions for estimating the electron density profiles are reasonable, which supports the idea 
that the electron density has been increasing with time in the past decades.

\section{Discussion}

\subsection{An ionized wind in CRL 618?}

Evidence of the increasing radio continuum emission in CRL 618 was reported for the first time by Kwok \& Feldman (1981). These authors showed that the flux doubled its value between 1977 and 1980. They explained this rapid rise of the radio flux as a result of the expaning ionization front into the neutral circumstellar envelope. In subsequent works it was reported that the increase of the radio flux seemed to have declined considerably, or even was halted (Kwok \& Bignell 1984; Martín-Pintado et al. 1988, 1993, 1995; Sahai et al. 1989). This was attributed to a deceleration of the ionization front (e.g. Spergel et al. 1983).

The emission flux of CRL 618 at mm wavelengths has also shown an increasing trend since 1977. A compilation of the flux at $\mathrm{mm}$ wavelengths as a function of time since 1977 until 2002 is presented in Table 2 of Sánchez-Contreras et al. (2004). In the plot of Fig. 6 we included the flux at mm wavelengths of four epochs. The flux exhibits an increasing trend for the first three epochs, but decreases in the last epoch (1998). MartínPintado et al. (1988) found that this source had increased its flux at $3.4 \mathrm{~mm}$ by a factor of 3.5 since 1977 . These authors pointed out that for a power-law distribution of the electron density, $\mathrm{n}_{\mathrm{e}} \propto r^{-2}$, the flux increase at mm wavelengths cannot be explained in terms of an expanding ionized region. Thus, they concluded that to explain the increasing flux density, one has to assume density inhomogeneities produced by changes in the mass-loss rate. Our observations also suggest that the density increased in the past. Now we show that the increasing electron density can also explain the growth of the major axis of the HII region of CRL 618.

We have shown that as of 2007 the flux and the size of the source at $22 \mathrm{GHz}$ have been continuously increasing. The radio images of CRL 618 at $5 \mathrm{GHz}$ taken in early 1980, as well as those obtained in this work (epoch 1998), show radio continuum emission extending by $\gtrsim 0^{\prime} .7$. This indicates that by the year 1980 the ionization front had expanded at least up to that distance. Therefore, the advance of the ionization front cannot explain the observed expansion of the major axis of the ionized region at $22 \mathrm{GHz}$, which extends only to $\lesssim 0 . ' 5$ (see Fig. 2). For the optically thick ionized region to expand, as our results indicate, it is necessary that its optical depth at larger radii increases with time. From (1) we see that the optical depth at a fiducial frequency on a line-of-sight path depends on the emission measure and the electron temperature. In Sect. 4 we found that assuming a constant electron temperature and depth of the source was a good approximation to explain the spectral index and the dependance of the major axis as a function of frequency. Furthermore, from our observations we found that the peak brightness of the emission does not vary by more than $10 \%$ from epoch 1982.48 to epoch 2007.52. For an optically thick region, the peak brightness depends only on the electron temperature. Therefore, we do not expect the electron temperature to have changed by more than $10 \%$ throughout the observing epochs. Thus, the contribution to the optical depth due to a variation of the electron temperature should be about $20 \%$. From Fig. 2 and Table 4 we see that minor axis increased by a factor of $\sim 1.8$ since the first epoch. If we assume that the depth of the source is equal to the minor axis, and since the emission measure is proportional to the depth of the source, we have that for a given line-of-sight path the emission measure should have increased by a factor of $\sim 1.8$ throughout the observing epochs. If we consider the line-of-sight paths that correspond to the edges of the source $\left(r_{2007.52}=0 . .5\right.$ and $r_{1982.48}=0$.'3) for the epochs 1982.48 and 2007.52, using our result that the electron density is proportional to $r^{-2.4}$, we have that the ratio of electron densities should be $(0.5 / 0.3)^{-2.4} \approx 0.3$. Thus, while the increase of the source depth accounts for a factor of 1.8 in the optical depth increase, the decrease of the density with distance introduces a factor of $0.3^{2}=0.09$, which results in an effective growth of $\Delta \tau_{v} \sim 20 \%$. From this we conclude that the increase of the minor axis and temperature alone cannot account for a significant increase of the optical depth, and consequently, the main factor responsible for the optical depth increase at a given distance from the star is the rise of the electron density.

From a detailed analysis of the central region of CRL 618 at optical wavelengths, Sánchez-Contreras et al. (2004) concluded that the gas in this region must be completely ionized. Therefore, the ionization fraction is practically unity and an increase of the ionizing photons does not result in an increase of the free electrons. The only way of increasing the electron density is by feeding it ionized gas with higher density. Thus, our results support the idea that CRL 618 is still expelling gas in the form of an ionized wind, whose mass-loss rate has been increasing with time. We used the equation of mass continuity to reproduce the electron density profile shown in Fig. 5. The best fit to our data gives an expansion velocity of the ionized gas of $\sim 22 \mathrm{~km} \mathrm{~s}^{-1}$ and an increase of the mass-loss rate from $\sim 4 \times 10^{-6}$ to $\sim 6 \times 10^{-6} M_{\odot} \mathrm{yr}^{-1}$ in the $\sim 130$ years previous to 1998 . This value of the mass-loss rate agrees with that obtained by Martín-Pintado et al. (1988) within a factor of two, once the distance to the source is scaled to $D=900 \mathrm{pc}$.

\subsection{Origin and morphology of the ionized wind}

The beginning of the ionization of the circumstellar envelope at this very early stage of the evolution of the post-AGB phase suggests that CRL 618 descends from a relatively high-mass star. The central star of CRL 618 has been classified to be spectral type B0 with a stellar temperature $T_{\star} \gtrsim 32000 \mathrm{~K}$ (Westbrook et al. 1975; Kaler 1978; Schmidt \& Cohen 1981). SánchezContreras et al. (2002) found evidence of CIII/CIV lines corresponding to the W-R bump, which is observed in the majority of carbon-dominated Wolf-Rayet-type, [WC], central stars of PNe. The presence of a [WC] central star might explain the high mass-loss rate observe in this source. It might also explain why the mass-loss rate was increasing during the last century but decreased recently. The [WC] stars of post-AGB objects are known to experience periods of intense mass-loss. It could be possible that the central star of CRL 618 underwent a period of high mass-loss followed by a decrease of the mass ejection. In about 1971 the electron density at the very base of the wind became low enough for the ionizing photons to escape, which ionized the entire wind. The sudden flux increase seen in the years before 1980 could have been due to the advancing ionization front. Once the wind was completely ionized, the increasing size of the HII region has been mainly due to the expansion of the higher electron density parts of the ionized wind at constant velocity. Therefore, we consider that we are witnessing the birth of a planetary nebula in CRL 618.

In Fig. 7 we present a radio image of CRL 618 at $43 \mathrm{GHz}$ (shown in contours) superimposed on an gray-scaled image at $8.4 \mathrm{GHz}$. The size of the synthesized beam of the $43 \mathrm{GHz}$ image is $\sim 0{ }^{\prime} 045$, which makes it the radio image of CRL 618 with 


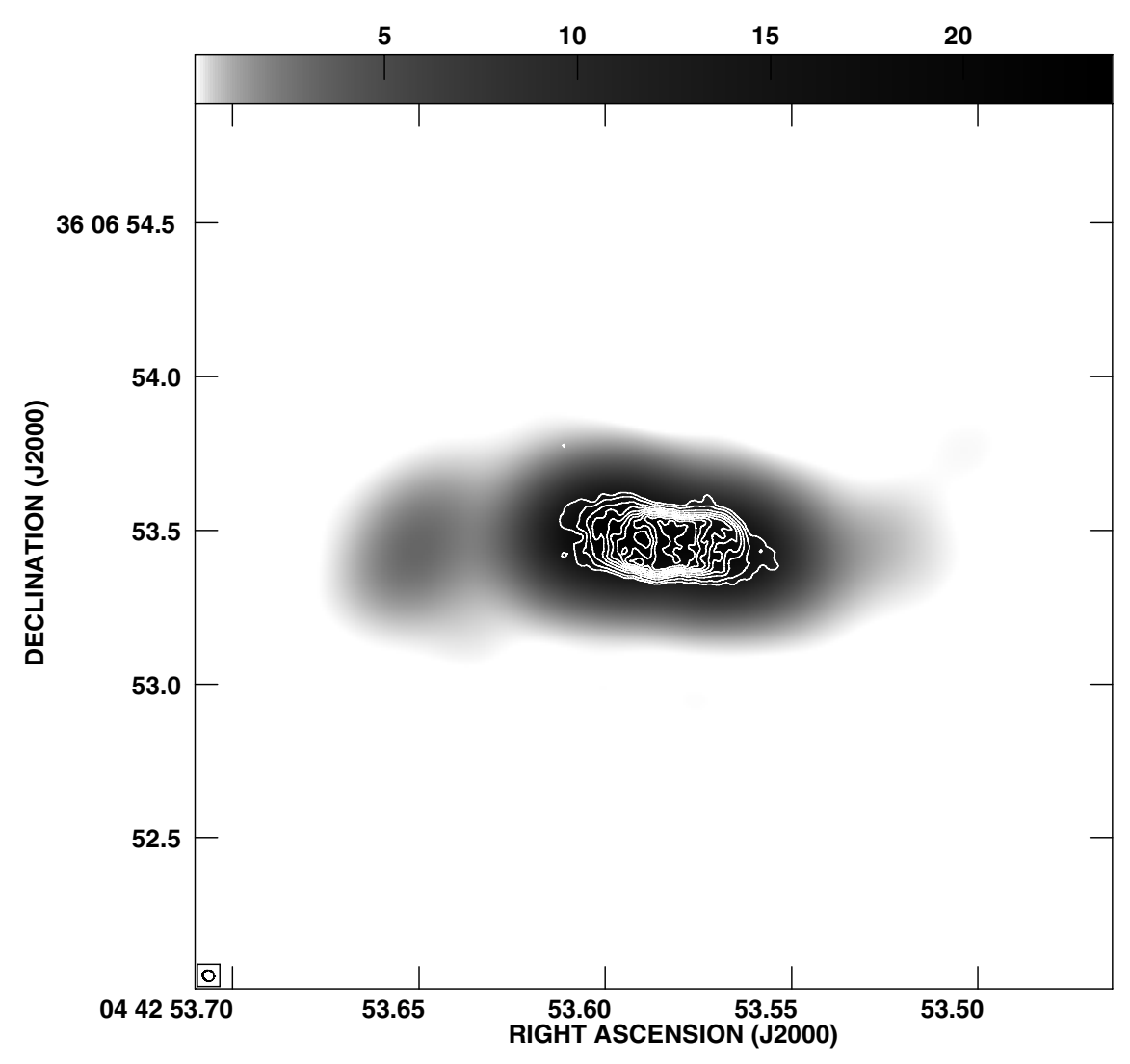

Fig. 7. Radio continuum emission of CRL 618 at $43 \mathrm{GHz}$ (contours) superimposed on a grayscale image of the radio continuum emission at $8.4 \mathrm{GHz}$. The values of the contours are $(-5,5,10,20,30,40,50,60,70,80,90$, $100) \times 2.4 \times 10^{-4} \mathrm{Jy}$ beam $^{-1}$, the rms noise of the image at $43 \mathrm{GHz}$. The size of the beam at $43 \mathrm{GHz}$ is $\sim 00^{\prime} 043 \times 00^{\prime} .046$ with $\mathrm{PA}=30^{\circ}$, and it is shown in the bottom left corner of the image. The gray-scale uses the logarithmic intensity transfer function $I^{\prime}=\log _{10}(1+9 \cdot I)$, where the intensity, $I$, covers the range of values from 0 to $2.38 \times 10^{-2} \mathrm{Jy} \mathrm{beam}^{-1}$. The size of the beam at $8.4 \mathrm{GHz}$, not shown in the image, is $\sim 0$.' $34 \times 00^{\prime} 28$ with $\mathrm{PA}=-71^{\circ}$. the highest angular resolution available so far. This image shows that the HII region has very well defined boundaries, parallel to each other, in the direction of the minor axis. The images at 5 , $8.4,15$ and $22 \mathrm{GHz}$ reveal that the source exhibits a subtle Sshape delineated by two faint extensions (see gray-scale image in Fig. 7). Particularly the extension toward the left appears to be disconnected from the main part of the HII region. This structure had not been detected previously, most likely due to the lack of sensitivity. Since it lies along the direction of the major axis, it might be density enhancement of the ionized stellar wind, suggesting that there has been an episode of discrete mass ejection. The S-shape morphology of the ionized region possibly hints at some kind of precessing mechanism, which would lead to the suggestion of a binary system. In fact, if one of the members of the binary system is a white dwarf and the other is a mass-losing AGB star, it might explain our observations in terms of the white dwarf ionizing the wind from the AGB star whose mass-loss rate had been increasing in the last century and just recently dropped.

Given the elongated morphology in the east-west direction of the wind, one might wonder whether this is because the wind is intrinsically collimated in this direction (i.e., a bipolar ejection), or that it is spherical, but suffers some kind of confinement along this direction. The optical images obtained with the Hubble Space Telescope reveal bullet-like structures located at the tips of the finger-like lobes. Even though they are located at different distances from the central star, Balick et al. (2013) have found that they share roughly the same kinematical age, $\sim 100$ years. Thus, it seems that around a century ago there was an event in which material was expelled with high velocities, creating cavities within the steadily expanding wind of the AGB phase. This event was followed by the onset of a stellar wind whose mass-loss was increasing with time until recently. In principle, the stellar wind does not need to be collimated intrinsically, but it expands preferentially in the direction of the cavities, because of the relative lower density in this direction. Thus, the full photoionization of the wind in the east-west direction seems to be directly related to the event that occurred $\sim 100$ years ago, which created the cavities. Had not these cavities been created, the ionization of the envelope would be constrained to a radius equal to the size of the minor axis in all directions. It seems that in the case of CRL 618 the configuration to form a bipolar planetary nebula was created only a century before the ionization of the CSE began. Therefore, the post-AGB phase of this source was extremely short.

\section{Conclusions}

We have traced the increase of the radio continuum flux at 5 and $22 \mathrm{GHz}$ for a period of $\sim 26$ years. The flux exhibits a monotonic growth with time up to 2007 . We measured the growth of the size directly from the brightness distribution of the emission. The major axis is expanding twice as fast as the minor axis. The increasing projected area of the ionized region corresponds to the increasing radio flux, indicating that the emitting region is basically optically thick. The ionization of the material around CRL 618 began some time around 1971. In the first years the growth of the ionized region was faster, and recently it became roughly linear. While the major axis of the ionized region exhibits a dependance on frequency, the size of the minor axis is the same at all observing frequencies. This behavior is explained in terms of an electron density gradient in the direction of the major axis. The HII region is ionization-bound in the direction of the minor axis, indicating a much higher density of the material in this direction. The density of the material in the direction of the major axis has increased in the last century, although in the recent decades it seems to have experienced a drop. The density decrease close to the central star explains the beginning of the ionization around 1971 and the observed expansion in the 
direction of the major axis. This event marked the beginning of the planetary nebula phase for CRL 618, implying a very short time for its post-AGB phase, $\sim 100$ years.

Acknowledgements. This research was supported by the Deutsche Forschungsgemeinschaft (DFG; through the Emmy Noether Research grant VL 61/3-1). L.L. acknowledges the financial support of DGAPA, UNAM and CONACyT, México. J.P.F. acknowledges the Spanish Ministerio de Educación y Ciencia for funding support through grants ESP 2004-665 and AYA 2003-2785 and the "Comunidad de Madrid" government under PRICIT project S-0505/ESP-0237 (ASTROCAM). This study has been supported in part by the UNAM through a postdoctoral fellowship and the European Community's Human Potential Program under contract MCRTN-CT-2004-51230, "Molecular Universe". The authors are grateful to Bruce Balick for valuable discussions and suggestions.

\section{References}

Altenhoff, W., Mezger, P. G., Wendker, H., \& Westerhout, G. 1960, Technical Report 59, Veröff. Univ. Sternwarte, Bonn, 48

Bachiller, R., Gomez-Gonzalez, J., Bujarrabal, V., \& Martín-Pintado, J. 1988, A\&A, 196, 5

Balick, B. 1987, ApJ, 94, 671

Balick, B., Huarte-Espinosa, M., Frank, A., et al. 2013, ApJ, in press

Beckwith, S., Persson, S. E., \& Gatley, I. 1978, ApJ, 219, L33

Bujarrabal, V., Gómez-González, J., Bachiller, R., \& Martin-Pintado, J. 1988, A\&A, 204, 242

Bujarrabal, V., Castro-Carrizo, A., Alcolea, J., \& Sánchez Contreras, C. 2001, A\&A, 377, 868

Calvet, N., \& Cohen, M. 1978, MNRAS, 182, 687

Calvet, N., \& Peimbert, M. 1983, Rev. Mex. Astron. Astrophys., 5, 319

Cernicharo, J., Guélin, M., Penalver, J., Martin-Pintado, J., \& Mauersberger, R. 1989, A\&A, 222, L1

Cernicharo, J., Heras, A. M., Tielens, A. G. G. M., et al. 2001, ApJ, 546, L123
Goodrich, R. W. 1991, ApJ, 376, 654

Gottlieb, E. W., \& Liller, Wm. 1976, ApJ, 207, L135

Hajian, A. R., Phillips, J. A., \& Terzian, Y. 1996, ApJ, 467, 341

Kaler, J. B. 1978, ApJ, 220, 887

Kastner, J. H., Gatley, I., \& Weintraub, D. A. 2000, Asymmetrical Planetary

Nebulae II: From Origins to Microstructures, 199, 355

Knapp, G. R., \& Morris, M. 1985, ApJ, 292, 640

Knapp, G. R., Sandell, G., \& Robson, E. I. 1993, ApJS, 88, 173

Kwok, S., \& Bignell, R. C. 1984, ApJ, 276, 544

Kwok, S., \& Feldman, P. A. 1981, ApJ, 247, L67

Lee, C.-F., \& Sahai, R. 2003, ApJ, 586, 319

Martín-Pintado, J., \& Bachiller, R. 1992, ApJ, 391, L93

Martín-Pintado, J., Bujarrabal, V., et al. 1988, A\&A, 197, L15

Martín-Pintado, J., Gaume, R., Bachiller, R., \& Johnston, K. J. 1993, ApJ, 419, 725

Martín-Pintado, J., Gaume, R., Johnston, K. J., \& Bachiller, R. 1995, ApJ, 446, 687

Neri, R., García-Burillo, S., Guelin, M., et al. 1992, A\&A, 262, 544

Pardo, J. R., Cernicharo, J., \& Goicoechea, J. R. 2005, ApJ, 628, 275

Phillips, J. P., Williams, P. G., Mampaso, A., \& Ukita, N. 1992, A\&A, 260, 283

Reuter, H.-P., Kramer, C., Sievers, A., et al. 1997, A\&A, 122, 271

Sahai, R., \& Trauger, J. T. 1998, ApJ, 116, 1357

Sahai, R., Claussen, M. J., \& Masson, C. R. 1989, A\&A, 220, 92

Sánchez-Contreras, C., \& Sahai, R. 2004, ApJ, 602, 960

Sánchez-Contreras, C., Sahai, R., \& Gil de Paz, A. 2002, ApJ, 578, 269

Sánchez-Contreras, C., Bujarrabal, V., Castro-Carrizo, A., Alcolea, J., \& Sargent, A. 2004, ApJ, 617, 1142

Schmidt, G. D., \& Cohen, M. 1981, ApJ, 246, 444

Spergel, D. N., Giuliani, Jr., \& Knapp, G. R. 1983, ApJ, 275, 330

Teräsranta, H., Tornikoski, M., Valtaoja, E. et al. 1992, A\&A, 94, 121

Trammell, S. R. 2000, ASPC, 199, 147

Walker, R. G., \& Price, S. D. 1975, Report AFCRL-TR-75-0373

Walmsley, C. M., Chini, R., Kreysa, E., et al. 1991, A\&A, 248, 555

Westbrook, W. E., Becklin, E. E., Merril, K. M., et al. 1975, ApJ, 202, 407

Wynn-Williams, G. G. 1977, MNRAS, 181, 61 